\title{
Les représentations des pratiques vidéoludiques dans Le Monde : entre loisir et problème de santé publique
}

The representations of the practices of videogame playing in Le Monde: between leisure and public health problem

\section{Audrey Arnoult}

\section{OpenEdition}

\section{Journals}

Édition électronique

URL : http://journals.openedition.org/sdj/2637

DOI : $10.4000 /$ sdj. 2637

ISSN : 2269-2657

\section{Éditeur}

Laboratoire EXPERICE - Centre de Recherche Interuniversitaire Expérience Ressources Culturelles Education

\section{Référence électronique}

Audrey Arnoult, « Les représentations des pratiques vidéoludiques dans Le Monde : entre loisir et problème de santé publique », Sciences du jeu [En ligne], 13 | 2020, mis en ligne le 11 novembre 2020, consulté le 21 janvier 2021. URL : http://journals.openedition.org/sdj/2637 ; DOI : https://doi.org/ 10.4000/sdj.2637

Ce document a été généré automatiquement le 21 janvier 2021.

\section{(c) $($ i) $(9)$}

La revue Sciences du jeu est mise à disposition selon les termes de la Licence Creative Commons Attribution - Pas d'Utilisation Commerciale - Pas de Modification 4.0 International. 


\section{Les représentations des pratiques vidéoludiques dans Le Monde : entre loisir et problème de santé publique}

The representations of the practices of videogame playing in Le Monde: between leisure and public health problem

Audrey Arnoult

$1 \quad$ Notre contribution étudie les discours sur l'addiction et les pratiques excessives du jeu vidéo dans le quotidien Le Monde. Le jeu vidéo peut être défini comme l'ensemble des jeux se réalisant à travers un dispositif informatique (ordinateur, smartphone, tablette, etc.) que celui-ci soit relié ou non à une connexion Internet. Le joueur pratique seul ou en groupe, dans un environnement virtuel (INSERM, 2014, p.11). Il en existe une grande variété : jeux d'application sur un smartphone, jeux d'actions en ligne, jeux de simulation ou encore jeux de rôle multijoueurs, également appelés Massively Multiplayers Online (MMO). Les jeux vidéo sont une "expérience instrumentée " (Triclot, 2011, p. 16) au même titre que le cinéma, la lecture ou encore la musique. En effet, les pratiques culturelles reposent sur des dispositifs techniques afin de «favoriser la production de certaines formes d'expériences» (Triclot, 2011, p. 16). Pourtant, les pratiques vidéoludiques suscitent régulièrement des débats quant à leur caractère violent, au risque d'addiction et à la confusion possible entre réel et virtuel.

D'un point de vue médical la $5^{\mathrm{e}}$ version du Manuel diagnostique et statistique des troubles mentaux a inclus en 2013, les troubles liés au jeux vidéo en ligne ( Internet Gaming Disorder ») dans la section consacrée aux troubles devant encore faire l'objet de recherches. Comme le souligne Bellevergue, l'absence d'un trouble reconnu de l'addiction aux jeux vidéo «n'évacue pas pour autant l'existence d'une problématique spécifique aux usages excessifs » (Bellevergue, 2017, p. 73). Plus récemment, en mai 2019 , le trouble du jeu vidéo (gaming disorder) a été défini et intégré dans la $11^{\mathrm{e}}$ édition de la Classification Internationale des Maladies, validée dans le cadre de la World Health Assembly. 
Politiquement, en France, la question des jeux vidéo a été intégrée au Plan national de mobilisation contre les addictions (2018-2022). Elle fait donc l'objet d'une prise en charge récente de la part des pouvoirs publics. Cependant, plusieurs instances se sont intéressées à cette problématique dès les années 2000. Ainsi, en 2008, le Sénat a publié un rapport intitulé Le phénomène addictif: mieux le connaitre pour le combattre. En 2014, l'Observatoire Français des Drogues et des Toxicomanies a publié une étude montrant qu'un élève sur huit avait un usage problématique des jeux vidéo (Obradovic et al., 2014, p. 4). ${ }^{1}$ Les pratiques vidéoludiques peuvent par conséquent être considérées comme un problème public au sens où elles sont actuellement, en France, un «enjeu de débat public et/ou d'intervention étatique » (Neveu, 1999, p. 42).

\section{Problématique, corpus et méthodologie}

Inscrite dans le champ des Sciences de l'Information et de la Communication, notre contribution entend étudier la médiatisation de l'addiction et des pratiques excessives des jeux vidéo dans le quotidien national Le Monde. Dans une perspective constructiviste, les médias sont un des acteurs sociaux qui met « en lisibilité le monde social » (Delforce, 1996, p. 28). Leurs discours sont sous-tendus par des conceptions et des grilles d'intelligibilité qu'il nous faut interroger car elles véhiculent des représentations qui participent de la discursivité sociale. En outre, les médias sont un acteur parmi d'autres de la configuration d'un problème public. Les jeux vidéo faisant l'objet d'une politique de santé publique relativement récente en France, nous pouvons nous interroger sur les discours tenus par la presse quotidienne nationale. Sur quels types de pratiques se focalise-t-elle? Quelles significations leur sont-elles attribuées? Quelles représentations de la figure du joueur sont-elles données à lire? S'agit-il d'un adolescent qui joue pour le plaisir, d'un jeune dépendant que sa pratique fait souffrir ou d'une période transitoire avant l'âge adulte? Enfin, peut-on repérer une évolution des représentations médiatiques qui témoignerait d'un nouveau paradigme pour penser les pratiques excessives?

5 Pour répondre à ces questions, nous analysons un corpus composé des articles du Monde, parus entre le 4 mai 1998 et le 14 mai 2018. Le choix de ce quotidien s'explique à la fois par sa place dans le champ journalistique de l'information et la volonté de disposer d'un matériau circonscrit pour réaliser une analyse qualitative pertinente. Le Monde est un journal centriste dit « de référence » (Eveno, 2004, p. 58), créé en 1944 par Hubert Beuve-Méry. Il fait partie des quotidiens ayant ouvert leurs colonnes à l'information scientifique et technique, dès les années $1980 .{ }^{2}$ La temporalité de notre corpus est fonction des contraintes d'archivage sur la base de données Europresse, ${ }^{3}$ mais est nécessairement longue pour permettre une étude diachronique des représentations.

6 Méthodologiquement, une approche quantitative permet de caractériser la médiatisation des jeux vidéo: thématiques principales, journalistes qui couvrent le sujet et rubriquage des articles. Ces différents éléments nous donneront des indications sur le cadrage dont font l'objet les pratiques vidéoludiques dans Le Monde. Puis, une analyse qualitative s'appuyant à la fois sur la sémiotique narrative greimassienne et l'analyse lexicale, permettra de caractériser de manière plus fine le traitement médiatique du sujet. L'approche narrative vise à repérer le(s) phase(s) du schéma narratif qui sont privilégiées dans les discours. Le schéma narratif se compose des 
phases de manipulation, de compétences, de performance et de sanction. Celles-ci organisent le récit, mais ne sont pas nécessairement toutes présentes dans un discours. Nous nous focalisons plus précisément sur les phases de performance et de manipulation. La phase de performance est une phase de transformation, de «faireêtre " qui permet à l'actant sujet de réaliser sa quête. Il s'agit ici des pratiques vidéoludiques. La phase de manipulation en est une au cours de laquelle le destinateur fait agir l'actant sujet. Le destinateur est « celui qui fait faire, c'est-à-dire qu'il exerce un faire visant à provoquer le faire du sujet » (Greimas, 1976, p. 23). Il peut s'agir d'une personne, mais aussi d'un objet ou d'une émotion. Dans le cadre du jeu vidéo, elle peut correspondre aux facteurs déclencheurs des problèmes d'addiction associés aux pratiques vidéoludiques. Cette analyse est menée conjointement à une analyse lexicale portant sur les termes employés pour désigner les joueurs, leurs pratiques et les risques éventuels. Nous étudions également les différentes figures que construisent les discours pour prévenir ces conduites. Avant de présenter les résultats de ces analyses, nous revenons sur quelques éléments de contexte concernant la place des jeux vidéo dans les loisirs adolescents et les représentations sociales qui leur sont associés.

\section{Une préoccupation croissante relative à la place des jeux vidéo dans les loisirs adolescents}

\section{Quelques chiffres sur le marché et la pratique des jeux vidéo}

7 En France, le marché du jeu vidéo représente un chiffre d'affaires de 4,3 milliards d'euros en 2017 (contre 3,64 milliards d'euros en 2016) (SELL, 2018, p. 4 et 11). Le Programme d'Étude sur les Liens et l'Impact des Écrans sur l'Adolescent Scolarisé indique, qu'en 2013-2014, sept Français sur dix (âgé de 6 à 65 ans) déclaraient avoir joué à des jeux vidéo dans les six derniers mois. Cette part est de $96 \%$ chez les 10-14 ans et de $89 \%$ chez les 6-9 ans. Parmi les élèves interrogés, 1 sur 8 aurait un usage problématique des jeux vidéo. Le joueur problématique se distingue par une pratique solitaire du jeu, à domicile, le plus souvent en ligne, de type MMO. L'intensité de la pratique vidéoludique est déterminée par différents niveaux d'utilisation : occasionnel, régulier, excessif et addictif. L'Inserm, quant à lui, estime la prévalence de l'usage problématique juvénile entre 2 et $12 \%$, selon les pays et les échantillons (INSERM, 2014, p.13). En 2014-2015, parmi les jeunes s'étant rendus aux Consultations Jeunes Consommateurs, $6 \%$ ont consulté pour une conduite addictive aux jeux vidéo (Obradovic, 2018, p. 40). Précisons toutefois que les données sur l'usage des jeux vidéo chez les adolescents en France - notamment dans leur dimension addictive - sont rares et varient en fonction des échantillons et des modalités de recueil de données. Pourtant, ils ne sont pas sans susciter des craintes qu'il s'agisse du corps médical, des politiques ou encore des parents.

\section{Trouble lié aux jeux vidéo : une catégorie nosologique controversée}

8 La notion d'addiction aux jeux vidéo ne fait pas consensus (Valleur, 2009, p.10; INSERM, 2014, p. 15), à la fois à cause de la diversité des jeux vidéo existants mais aussi de la pluralité des méthodes et des échantillons mobilisés dans les études épidémiologiques. 
9 Dans le champ scientifique, l'Organisation Mondiale de la Santé a introduit le gaming disorder dans la $11^{\mathrm{e}}$ version de la Classification Internationale des Maladies (CIM). Ses caractéristiques sont les suivantes: le comportement de jeu est persistant ou récurrent ; le joueur éprouve des difficultés à contrôler les périodes de jeu ; une priorité accrue est accordée au jeu par rapport aux autres centres d'intérêt; la pratique se poursuit - voire s'intensifie - en dépit de ses conséquences négatives et les sphères personnelle, familiale et sociale sont délaissées. L'ensemble de ces critères doit être observé pendant au moins 12 mois pour que le diagnostic puisse être posé. La perspective d'intégrer le gaming disorder dans la CIM a suscité des réticences qui ont donné lieu à une lettre ouverte (Aarseth et al., 2017). Un ensemble d'experts ont souligné leur désaccord, critiquant les études existantes.

Dans le corps médical, certains médecins et psychiatres préfèrent parler de pratique excessive, celle-ci prenant souvent fin avec l'entrée dans la vie adulte. En outre, ils rappellent l'importance de distinguer les circonstances du jeu, les types de jeu et les profils des joueurs. En effet, l'addiction aux jeux (notamment en réseau) ne peut échapper "à la nécessaire approche "trivariée" popularisée par Olievenstein en matière de toxicomanies: là aussi, il s'agit d'une rencontre entre un produit, une personnalité, et un moment socioculturel » (Valleur, 2009, p.16). C'est pourquoi ce psychiatre invite à distinguer les "demandes "symptômes" " des "vrais cas de dépendance» (Valleur, 2006, p. 125). Il ne faut pas non plus oublier d'inscrire cette question dans une réflexion sur la fonction du jeu dans l'économie psychique du sujet : quel est l'intérêt du jeu pour l'adolescent ou le jeune adulte? Dans quelles mesures lui permet-il d'exprimer sa singularité ?

11 Politiquement, si les pouvoirs publics français se sont saisis de cette question relativement récemment, ce sujet est débattu depuis plusieurs années dans le champ politique. Par exemple, dans le rapport du Sénat de 2008 - mentionné en introduction nous pouvons lire que «l'addiction aux jeux vidéo n'est pas plus dangereuse qu'une autre forme d'addiction. Mais elle ne l'est pas moins ». Les rapporteurs appellent à ne «pas diaboliser cette nouvelle tendance » car « le plus souvent les enfants délaisseront à l'âge de l'adolescence leur écran d'ordinateur, pour d'autres conquêtes ». Pour autant, ils précisent $\mathrm{qu}^{\prime}$ «il ne faut pas être aveugle aux risques qu'ils comportent » (Sénat, 2008). Ces risques tiennent une place non négligeable dans les critiques adressées aux jeux vidéo et les représentations qui y sont associées.

\section{Quelques représentations sociales du joueur : entre dépendance et compétences}

12 Les craintes relatives aux jeux vidéo concernent le plus souvent la désocialisation, les comportements violents et la perte de repères qui serait liée à une confusion entre réel et virtuel. Ainsi, l'Inserm évoque la "perte de contrôle» et le "comportement problématique » que peut entraîner la pratique des jeux vidéo, notamment en réseau (INSERM, 2014, p. 11 et 50). Nachez et Schmoll alertent sur la «figure désormais popularisée par les médias, le fana de jeux vidéo, de plus en plus internaute, passe des heures devant son écran, ne sort plus de chez lui pour rencontrer ses parents, ses voisins et ses amis » (Nachez et Schmoll, 2003, p. 6). La désocialisation est également évoquée dans le plan national de mobilisation contre les addictions et les écrans. Pour les auteurs, «les jeux vidéo présentent des risques d'enfermement des jeunes dans des 
scénarios addictifs, irréels et parfois violents » (MILDECA, 2018, p. 27). La littérature scientifique mais aussi les médias seraient en partie à l'origine de la représentation du jeu vidéo «comme un objet pernicieux favorisant les comportements violents et l'addiction » (Bellevergue, 2017, p. 72). Issus de la littérature scientifique et de rapports politiques, ces quelques exemples témoignent d'une représentation stéréotypée du joueur dépendant.

13 En contrepoint, la pratique des jeux vidéo constitue un facteur d'intégration sociale. Courant à l'adolescence, ce loisir permet le développement d'une sociabilité hors ligne et en ligne. En outre, les jeux sont vecteurs d'apprentissage de connaissances, de savoir-faire et savoir-être. De la sorte, dans son analyse des types d'apprentissage permis par le jeu Dark Age of Camelot, Berry montre que pour évoluer dans l'univers du jeu, l'internaute doit connaître la géographie, la faune, la flore mais également des fondamentaux économiques. En outre, il doit développer des savoir-faire liés aux tactiques de combat (2007, p.79). Ces apprentissages se font majoritairement par l'intermédiaire des guildes, "transmetteur de la pratique» (2007, p. 80). De plus, contrairement aux séries télévisées, la pratique des jeux vidéo exige une conduite active: les décisions à prendre pour évoluer dans le jeu exigent des capacités de raisonnement, de négociation, de communication, etc. Enfin, le jeu est aussi «le lieu d'expression d'émotions qui sont réprimées dans le contexte social» (Nachez \& Schmoll, 2003, p. 8).

14 Ces représentations du jeu vidéo participent de la discursivité sociale autour de ce sujet. Les médias, acteurs sociaux, s'en saisissent pour les appuyer, ou les nuancer, voire les infirmer. Trouve-t-on des traces de ces représentations dans les discours médiatiques?

\section{Une analyse quantitative de la couverture médiatique du jeu vidéo}

15 Une analyse quantitative des articles publiés entre 1998 et 2018 permet d'éclairer la « mise en visibilité » (Delforce, 1996, p. 28) des pratiques vidéoludiques. Au préalable, nous explicitons les critères de constitution du corpus. Nous poursuivons avec l'analyse des caractéristiques de la couverture médiatique des pratiques vidéoludiques et l'étude des journalistes qui couvrent le sujet.

\section{Les critères de constitution du corpus}

16 Un corpus « n'a de sens, de valeur et de pertinence qu'au regard des questions qu'on va lui poser, des réponses que l'on cherche, des résultats que l'on va trouver » (Mayaffre, 2002). Nous voulons ici étudier le traitement médiatique des problématiques d'addiction associées aux pratiques vidéoludiques. Pour cela, nous avons constitué un corpus à partir de la base de données Europresse. La recherche a été effectuée avec plusieurs requêtes ${ }^{5}$, dans l'ensemble des archives du quotidien Le Monde - soit depuis 1944, jusqu'au 28 février 2019.

17 Nous avons obtenu plusieurs centaines d'articles en réponse à nos différentes requêtes. Une première lecture a permis d'exclure les articles sans rapport avec la thématique choisie. Nous avons également écarté les discours qui ne portaient pas sur la situation 
française. Les articles qui envisageaient les aspects commerciaux, économiques ou encore technologiques des jeux vidéo n'ont pas été inclus. Notre corpus final compte 26 articles, parus entre le 4 mai 1998 et le 14 mai 2018. Il satisfait donc au critère d'homogénéité. En outre, il répond au critère de diachronicité, l'objectif étant d'étudier la «mise en visibilité » et en "lisibilité » (Delforce, 1996, p. 28) des problématiques d'addiction associées aux pratiques vidéoludiques dans la presse, il était nécessaire de disposer d'une période assez longue pour repérer l'évolution des représentations.

\section{Quelques caractéristiques de la médiatisation des problématiques d'addiction associées aux pratiques vidéoludiques}

Les pratiques vidéoludiques sont médiatisées dans les colonnes du Monde dès la fin des années 1970. Le recueil - puis la sélection et la lecture du corpus - a permis de remarquer que c'est alors essentiellement dans une perspective économique et technologique qu'ils sont évoqués. Puis, les termes "dépendance " et "addiction » commencent à être associés aux jeux vidéo. Cependant, il s'agit dans un premier temps d'une réflexion assez générale sur la dépendance à Internet et l'impact des images. Le sujet de l'«addiction» aux jeux vidéo ne s'autonomise que dans les années 2000 puisque le premier article sur ce sujet paraît le 4 mai 1998. La production médiatique est très hétérogène et relativement faible, comme le montre le graphique ci-dessous :

Figure 1

Répartition des articles entre 1998 et 2018

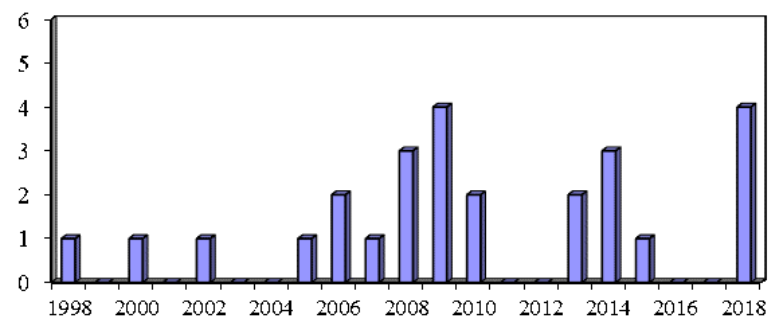

口Nombre đ'articles publiés

Selon les années, entre 0 et 4 articles sont publiés. La couverture médiatique de l'«addiction » aux jeux vidéo n'est pas rythmée par des événements spécifiques, à l'exception de deux articles portant sur la parution d'enquêtes scientifiques en 2008 (23/01/08) et 2014 (Santi, 18/12/04) Il n'y a donc pas de périodes distinctes dans la «mise en visibilité » de cette problématique dans les discours de presse. Cette faible médiatisation peut s'expliquer, entre autres, par l'absence de politique de santé publique spécifique aux jeux vidéo - jusque très récemment - et par l'incertitude scientifique et médicale entourant la notion d'addiction aux jeux vidéo. De ce fait, les journalistes ne disposent pas de cadrage pour appréhender le sujet. L'analyse du rubriquage confirme cette hypothèse. 


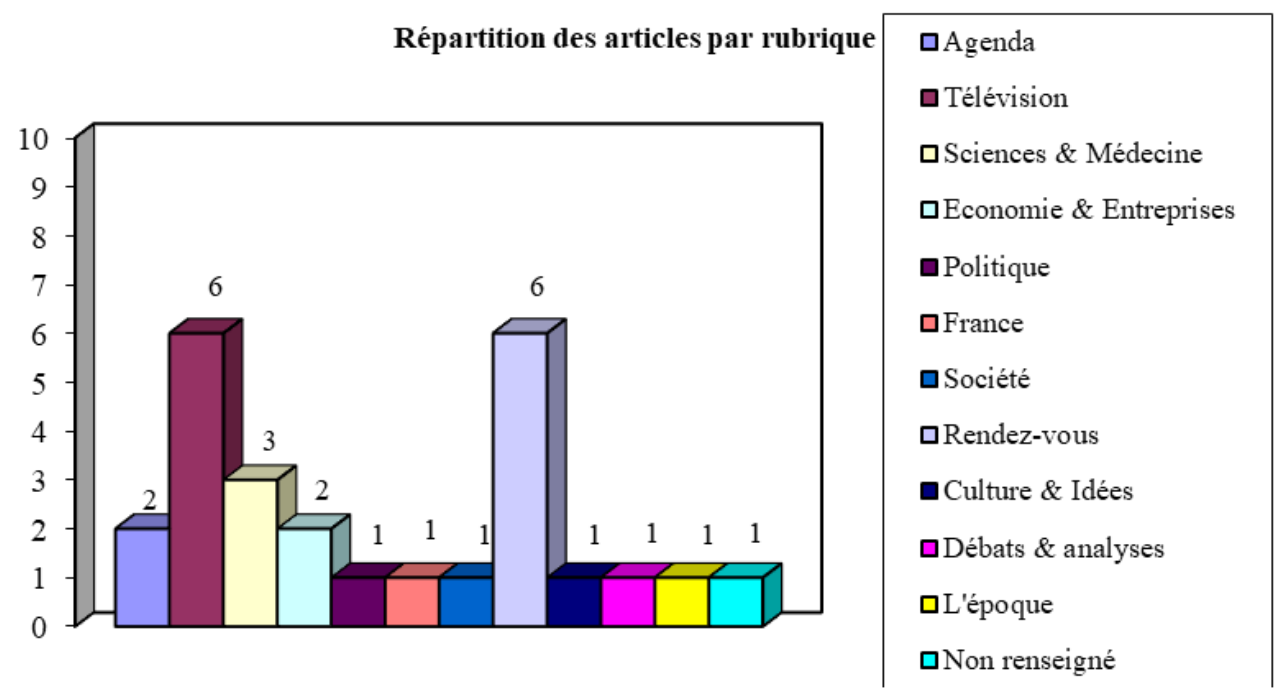

Le rubriquage est un choix déjà signifiant dans le cadrage d'un sujet. En effet, le langage « informe, certes, mais surtout construit du sens, des significations et cela dans une mise en scène qui elle-même contribue à l'édification du sens" (Chabrol, Charaudeau et Houdebine, 1988, p. 6). Les articles sont éparpillés dans l'ensemble des pages du journal. Il n'y a pas de «migration» (Moirand, 2001, p. 101-102) des articles des pages scientifiques du quotidien vers les autres rubriques, qui serait l'indicateur d'un traitement plus social et politique et donc de la constitution du sujet en problème public. Les rubriques «Télévision» et «Rendez-vous» sont celles qui recueillent «le plus » d'articles (6). Enfin, nous pouvons noter que seuls deux articles relèvent de la rubrique "Sciences \& Médecine », ce qui peut traduire le flou autour de la catégorie nosologique définissant ces pratiques.

\section{Les journalistes qui couvrent le sujet}

21 L'analyse du domaine de spécialité des journalistes qui signent les articles sur les problématiques d'addiction associées aux pratiques vidéoludiques permet de préciser les résultats précédents. Les 26 articles sont écrits par 16 personnes différentes, seules 4 signant plusieurs articles: Catherine Vincent (3), Martine Laronche (4), Marlène Duretz (2) et Pascale Santi (2). La thématique des pratiques vidéoludiques n'est pas couverte par quelques journalistes spécialisés sur le sujet. Catherine Vincent, Martine Laronche, ${ }^{6}$ Pascal Santi et Sandrine Cabut $^{7}$ sont journalistes scientifiques ou spécialisés dans le domaine de la santé, Nathalie Brafman - qui signe un article - est cheffe du service Société. Les autres signataires prennent en charge soit les contenus culturels ou multimédias, ${ }^{8}$ et seul un journaliste (William Audureau) est spécialisé dans le jeu vidéo. Cette diversité de journalistes qui couvrent le sujet témoigne des différents cadrages dont les pratiques vidéoludiques font l'objet.

L'analyse quantitative montre que la presse quotidienne accorde peu de visibilité au sujet de l'addiction aux pratiques vidéoludiques: aucun pic événementiel n'est repérable et les récentes mesures adoptées par les pouvoirs publics n'entraînent pas une augmentation de la production discursive. Au vu de ces résultats, nous pouvons nous interroger sur la façon dont cette thématique est mise en discours par le journal. 


\section{La « mise en lisibilité » des pratiques vidéoludiques: une " addiction » qui fait débat}

Nous étudions la « mise en lisibilité » de l'addiction aux pratiques vidéoludiques et plus précisément la façon dont le journal construit (ou non) cette thématique comme un problème de santé publique en nous focalisant sur trois éléments : la désignation des joueurs et de leur activité, la représentation des pratiques et de leurs conséquences, la place accordée à la prévention et/ou la prise en charge.

\section{La désignation des joueurs et de leur « addiction »}

Les critères de constitution de notre corpus induisent une forte homogénéité dans le contenu des discours et plus précisément sur le type de joueurs dont il est question. La plupart des articles portent sur la question de l'existence - ou non - d'une addiction aux jeux vidéo chez les adolescents, de ses conséquences, de sa prise en charge et dans une moindre mesure - de la prévention à mettre en œuvre. Seuls trois articles évoquent la pratique des jeux vidéo dans l'ensemble de la population française et chez les seniors. ${ }^{9}$ Le Monde se focalise donc principalement sur les jeunes joueurs ayant une pratique excessive. Ce choix peut s'expliquer par l'attrait du virtuel chez les jeunes et la sociabilité que permettent les jeux vidéo, le groupe de pairs jouant un rôle primordial à cette période de la vie.

L'actant sujet des pratiques vidéoludiques est, le plus souvent, un adolescent ou un jeune adulte, désigné par les termes "élève[s]», «adolescent $[\mathrm{s}]$ », "garçon[s] », « fille[s] », « jeune[s] homme[s]» ou encore " enfant $[s] »{ }^{10}$ Ce dernier substantif est parfois accompagné d'un qualificatif qui met en avant leur jeunesse (des "enfants, parfois très jeunes »-Vincent, 4/05/05), une façon d'alerter sur la situation. La distinction fille/garçon est employée quand le quotidien cite des enquêtes épidémiologiques distinguant les pratiques en termes de fréquence, de types de jeu et de genre. Nous trouvons également des substantifs qui condensent la performance ${ }^{11}$ tels que « joueurs » ou «gamers». L'ensemble de ces termes est relativement neutre et ne stigmatise pas le joueur. En revanche, deux articles évoquent la figure du « hardcore gamer» et du "no life» (Brafman, 11/05/06; Fiévet, 5/03/08). Ces désignations comportent implicitement une évaluation quantitative des pratiques vidéoludiques : ce sont des personnes dont la vie s'organise autour du jeu. En effet, la figure du "no life » est née au moment de la diffusion des jeux de rôle sur Internet. Ainsi, elle "a d'abord désigné des "accros", des "addicts" ou des passionnés. Il s'agissait souvent d'adolescents ayant des comportements inhabituels d'exclusion de leur groupe social » (Fouchard, 2013, p. 79). Toutefois, ces désignations ne comportent pas de jugement de valeur contrairement au terme de "drogué ", présent à deux reprises dans un article. L'apparition d'une "nouvelle catégorie de drogués aux jeux sur écran " (Vincent, 3/05/06) est avancée. Si l'existence de cette catégorie est finalement niée, le terme renvoie l'image d'une personne désocialisée, qui ne peut vivre sans sa "substance». Enfin, les expressions «jeunes patients» (Vincent, 4/05/05) et "jeunes accros» (Fiévet, 5/03/08) situent les joueurs du côté de la pathologie et de la souffrance. Certaines pratiques vidéoludiques peuvent donc nécessiter une prise en charge. 

personnalisation. Effectivement, dans certains articles, les joueurs sont désignés par leur nom et leur âge. Cette personnalisation donne un visage aux joueurs des pratiques vidéoludiques qui peuvent être des enfants, des adolescents mais aussi de jeunes adultes. Notons que seuls deux enfants de 6 et 8 ans sont présents dans notre corpus. Environ la moitié des joueurs dont l'âge est mentionné sont de jeunes adultes : Le Monde parle de patients de " 17 à 25 ans ", de jeunes hommes hospitalisés de 21 et 22 ans, de Paul un «jeune homme de 25 ans », d'« un jeune Isérois de 24 ans », de Simon qui a 35 ans, etc. L'autre moitié est constituée de mineurs: les «13-15 ans»; Charles, 16 ans tout comme Lola et Lisa ; Nino, 17 ans ou encore deux adolescents de 11 et 13 ans. Dans les discours du quotidien, la figure du joueur est principalement celle d'un adolescent ou d'une jeune adulte. Qu'en est-il de leurs pratiques? Les décrit-on comme des joueurs occasionnels, excessifs ou repliés sur eux-mêmes et leurs jeux vidéo?

\section{Les représentations des pratiques du jeu vidéo : une carrière de l'addiction?}

Il s'agit de voir comment les discours médiatiques construisent la figure d'un joueur dépendant au détriment d'autres discours médiatiques présentant le jeu vidéo comme un loisir parmi d'autres. En outre, nous pouvons nous demander si, dans ces articles portant sur les problèmes d'addiction associés aux jeux vidéo, nous trouvons malgré tout des représentations positives des pratiques vidéoludiques ou si Le Monde se focalise essentiellement sur les risques de ce loisir. Nous recourons ici au concept sociologique de "carrière $»^{12}$ pour analyser les pratiques des joueurs. Cette notion, empruntée à Becker, permet de penser la performance de façon séquentielle : jouer aux jeux vidéo ne conduit pas inéluctablement à la dépendance. Plusieurs niveaux de pratique existent. L'objectif est est de voir sur quelle(s) phase(s) se focalise Le Monde (le jeu occasionnel, régulier, excessif ou dépendant).

La phase de la performance est présente dans notre corpus de plusieurs façons. Elle est parfois réduite aux verbes «jouer» ou «s'adonner», parfois accompagnés d'un adverbe ou d'une expression qui indique la phase de la carrière dans laquelle se trouve le joueur. Par exemple : « un enfant qui ne travaillait plus en classe et passait son temps à jouer sur des jeux en réseaux » (Kerviel, 11/05/09) ou encore, « du milieu de l'aprèsmidi jusqu'au cœur de la nuit, on le retrouve chaque jour devant son ordinateur " (Delahaye, 3/08/09). Ces extraits renvoient à une pratique excessive, voire addictive. C'est parfois l'adjectif qualifiant le mot « joueur » qui nous renseigne sur la phase de la carrière: "joueurs très excessifs", "cyber addicts ", "gosses addicts " ou encore «accros aux jeux vidéo ». Dans un autre article, un psychiatre évoque les patients qui viennent le consulter en ces termes: "ils n'arrivent plus à s'arrêter de jouer " (Vincent, 4/05/05). La perte de liberté qui est suggérée constitue la caractéristique principale de l'addiction. La pratique est ici non maîtrisée.

29 À côté de cette représentation assez succincte de la performance du joueur, nous avons relevé plusieurs témoignages qui retracent la carrière de gamers. Simon, « vingt-cinq ans d'expérience des jeux », est qualifié de "hardcore gamer »: «il joue cinq jours par semaine entre quatre heures et cinq heures quotidiennement " (Brafman, 11/05/06). Son témoignage montre un joueur intégré professionnellement mais qui regrette que ses horaires de travail ne lui permettent pas de se connecter plus tôt. La pratique 
vidéoludique est régulière mais ne s'intensifie pas, il n'y a pas d'addiction. Le témoignage d'un psychiatre va dans ce sens. Il souligne qu'à «l'adolescence, tout est flottant, rien n'est jamais fixé. Il n'est pas rare de voir des joueurs très excessifs en troisième et seconde qui ne le sont plus en première ou en terminale " (Laronche, 7/01/09). L'accent est mis sur la phase de transition que constitue l'adolescence. Un comportement adopté à cette période de la vie ne se pérennise pas forcément, un joueur très excessif pouvant diminuer sa pratique.

30 En revanche, Marc Landré, 22 ans, « se souvient du processus qui l'a rendu dépendant aux jeux vidéo » (Laronche, 22/03/10). Le mot "processus» connote une évolution inéluctable, sur laquelle Marc n'a pas de prise. Il indique avoir joué « parfois jusqu'à 20 heures par jour ». Son inscription dans un internat lui permet de sortir de l'addiction. Aujourd'hui, il est un joueur "raisonnable», professionnellement intégré, mais il « assure » que «jamais [ses] enfants n'auront d'ordinateur dans leur chambre ». Nous trouvons des éléments similaires dans le troisième témoignage d'un joueur pour lequel les jeux vidéo sont "clairement une drogue ». "No life ", il passe son temps à jouer précisant « le dernier mois, j'étais au chômage, j'ai joué dix heures par jour. WOW ${ }^{13}$, ça aide pas à trouver un boulot » (Brafman, 11/05/06).

\section{Les risques des pratiques vidéoludiques : des risques controversés}

Les risques liés aux pratiques vidéoludiques sont un élément important dans les débats sur les jeux vidéo. Il s'agit donc de repérer les risques qui leur sont associés et dans quelles mesures ces pratiques sont présentées comme pouvant engendrer une addiction. L'analyse montre que deux types de représentations coexistent dans notre corpus: d'un côté, certains journalistes construisent la représentation de pratiques conduisant à la désocialisation, la violence et l'addiction; de l'autre, ce sont la sociabilité et les effets positifs des jeux vidéo qui sont mis en avant.

Dans neuf articles, les pratiques vidéoludiques sont présentées comme problématiques, entraînant le repli sur soi voire la désocialisation de l'adolescent ou du jeune adulte. Par exemple, à propos de deux jeunes hospitalisés en service psychiatrique " pour avoir abusé des jeux vidéos", une journaliste écrit "au fil des mois, ils se sont progressivement déconnectés de toute vie familiale et sociale et engloutis dans l'univers parallèle d'un "jeu de rôle en ligne massivement multi-joueurs" » (Vincent, 3/05/06). Le terme "engloutis » connote la perte de liberté et l'absence de maîtrise, caractéristiques de l'addiction. C'est aussi la désocialisation qui est avancée par un membre du Forum des droits sur Internet: «A jouer trop longtemps, les joueurs courent le risque de perdre tout lien social » (Ducourtieux, 25/12/07). Un gamer en témoigne : «C'est clairement une drogue. Il m'est arrivé plusieurs fois de refuser de sortir avec des amis parce que j'allais jouer» (Brafman, 11/05/06). Des parents racontent aussi la «crise» de leur enfant quand il ne peut pas jouer et sa "perte d'intérêt pour les autres activités " (Laronche, 23/01/13). Or, le désinvestissement des sphères familiale et amicale ainsi que le délaissement d'activités auparavant appréciées, constituent les critères d'une conduite addictive. La question de la violence apparaît dans quelques discours et fait l'objet d'un article en particulier, mais sans lien explicite avec l'addiction. En effet, un paragraphe introductif donne quelques chiffres sur les pratiques vidéoludiques puis plusieurs questions sont posées au professeur de psychologie sociale Laurent Bègue sur les «jeux vidéo violents ». Par exemple, nous 
pouvons lire que «les jeux vidéo violents ont une incidence sur les affects, les pensées et les conduites agressifs, et diminuent les conduites coopératives" (29/10/14). La construction de l'article sous-entend implicitement plus le temps passé à jouer est important, plus ces effets sont graves. Enfin, outre la désocialisation, les comportements agressifs et la perte d'intérêt pour d'autres activités, plusieurs articles soulignent le potentiel addictif des jeux vidéo ou constatent une addiction déjà-là : «l'addiction de Johan », « les dangers de l'addiction aux jeux vidéos [sic]»; chez les $16 / 25$ ans, les jeux vidéo " peuvent provoquer des addictions graves », etc.

Cette représentation des risques des pratiques vidéoludiques coexiste avec une autre représentation, principalement lisible dans les propos d' « experts » ${ }^{14}$ auxquels Le Monde donne la parole. Ils s'accordent sur les effets positifs des jeux et remettent en question l'existence d'une addiction. Ainsi, pour Marc Valleur (psychiatre), les craintes de violence et de confusion entre réel et virtuel sont "en grande partie infondées " (Vincent, 4/05/05). Les jeux vidéo sont source de " plaisir » (Vincent, 4/05/05; Vincent, $3 / 05 / 06$ ) et d'« enrichissement » (Vincent, 3/05/06). Ces arguments font écho à ceux de Vincent Berry, sociologue du jeu ou encore de Serge Tisseron, psychiatre. Pour le premier, l'isolement par l'écran est un "mythe», le jeu vidéo s'inscrivant " complètement dans les sociabilités des ados [...]. Les jeunes s'invitent et jouent ensemble autour de la console. Ou alors ils jouent en réseau, ce qui est une autre façon de ne pas être seul » (Vincent, 24/05/14). En outre, les jeux vidéo ne rendent pas les joueurs plus agressifs mais sont «le plus souvent des solutions imaginaires que des problèmes" (Vincent, 24/05/14). Les cas graves et pathologiques sont, pour lui, exceptionnels. De même, pour Serge Tisseron, le repli sur soi n'est pas la conséquence d'une pratique trop intense mais un facteur déclencheur : «c'est parce qu'ils éprouvent le besoin de se replier sur eux-mêmes que les jeunes sont conduits à jouer de manière excessive» (Laronche, 7/01/09). À l'inverse, pour le psychiatre, les jeux vidéo favorisent la socialisation notamment quand ils sont en réseau. Ils permettent aussi " de mettre en scène leur conflit conscient et inconscient " (Laronche, 7/01/09). Enfin, Vincent Berry rappelle qu'ils sont « une culture en soi » au même titre que la lecture ou la musique. Cette dimension culturelle est peu présente dans le corpus mais doit néanmoins être soulignée. ${ }^{15}$

Logiquement, les «experts» cités précédemment s'accordent pour considérer l'addiction aux jeux vidéo "comme un phénomène rare" (Serge Tisseron, pédopsychiatre interviewé par Laronche, 07/01/09). Dans un autre article, le quotidien titre "Jeux vidéo: une pratique répandue où les phénomènes de dépendance sont mineurs » (Laronche, 22/03/10) . Le terme de « joueur excessif » est privilégié par Serge Tisseron. Valleur quant à lui rappelle qu'il est important de prêter attention à la conduite du joueur dans son ensemble. En effet, dans la prise en charge de la toxicomanie, l'approche centrée sur le produit a laissé place à une approche focalisée sur le comportement (Valleur et Velea, 2002, p. 2). Ce n'est donc pas uniquement la nature de la substance ou du comportement qui doit être prise en compte pour déterminer si l'usage est " pathologique » mais le rapport du consommateur au produit ou au comportement (Bailly, 2009, p. 88). Enfin, il est rappelé qu'il existe «très peu de données sur les troubles réels qu'ils engendrent sur le comportement psychique, mental et social des enfants » (Zilbertin, 2/06/08). 


\section{Les facteurs déclencheurs des problématiques d'addiction aux pratiques vidéoludiques}

Les facteurs déclencheurs des problématiques d'addiction aux pratiques vidéoludiques sont peu évoqués. Ils ne font pas l'objet d'un article en particulier mais nous trouvons des éléments épars dans plusieurs discours. La figure du destinateur renvoie à la fois à des éléments de vie, à la personnalité de l'adolescent et - dans une moindre mesure au contenu du jeu et au rôle des parents. La dépendance s'installe lors de "phases existentielles difficiles" associées à une "situation familiale difficile» (Vincent, $3 / 05 / 06)$. Nous pouvons également lire que le jeu en excès prend place " au cours de certaines périodes de vie ", comme la séparation des parents ou un décès (Laronche, 7/01/09; Laronche, 22/03/10). Ce contexte de vie peut être associé à un certain type de personnalité. Ainsi, un psychiatre évoque des patients «introvertis, timides ou phobiques» (Vincent, 4/05/05). Ailleurs, ce sont des adolescents "fragiles», qui ont une faible estime d'eux-mêmes. Les pratiques problématiques sont alors le «symptôme d'une souffrance sous-jacente » (Laronche, 11/10/10). Notons que cette souffrance n'est évoquée que dans deux articles. Le prisme de la pathologie est donc mineur dans la représentation des pratiques vidéoludiques excessives. Le troisième facteur de l'addiction serait contenu dans le jeu en lui-même. Cette idée ne figure qu'à trois reprises dans notre corpus. Ainsi, certains jeux reposent sur "une règle du jeu très simple, un peu de violence mais pas trop, la possibilité de jouer seul ou à plusieurs » (Brafman, 11/05/06). Dans un autre article, Serge Tisseron évoque les jeux en réseaux type World of Warcraft, "particulièrement préoccupant " car «le jeu ne s'arrête jamais » (Laronche, 7/01/09). Les jeux en réseaux seraient « conçus pour être addictifs, c'est-à-dire pour rendre dépendant» (Duretz, 3/04/13). Notons que considérer un jeu comme addictif relève d'une approche centrée sur le produit qui exclut l'environnement et la personnalité du joueur. Enfin, le dernier facteur explicatif d'un abus de jeux vidéo concerne l'attitude des parents. Cet élément apparait à deux reprises. Marc Valleur, psychiatre, estime que lorsque de jeunes adolescents jouent trop mais sans être dans la dépendance, il s'agit d'un "problème de guidance parentale » (Laronche, 11/10/10). De façon identique, une enquête ${ }^{16}$ souligne que « la pratique excessive du jeu vidéo est souvent liée à un défaut de surveillance et de sollicitude parentale »(Santi, 18/12/14).

\section{Les figures de la prévention d'une pratique addictive}

Ce dernier axe de l'analyse s'intéresse aux figures construites par les discours pour prévenir et/ou prendre en charge les pratiques vidéoludiques excessives. La figure des parents est la plus présente alors que celle des pouvoirs publics est quasi-absente.

\section{Les parents : un acteur au triple rôle actantiel}

Dans notre corpus, la figure des parents recouvre trois rôles actantiels : celui d'antidestinateur, d'anti-sujet et - dans une moindre mesure - de destinateur d'un nouveau programme narratif. En effet, les parents sont d'abord ceux qui posent des limites afin de réguler la pratique de leur enfant. Ils préviennent ainsi un usage qui pourrait devenir problématique. Ils " doivent être attentifs aux normes $"{ }^{17}$ ils " doivent être 
vigilants » (Vincent, 3/05/06), "s'inquiéter quand les résultats scolaires de leur enfant baissent " (Laronche, 7/01/09), "délimiter un cadre" (Duretz, 14/05/18), etc. Cependant, les parents ne se contentent pas de fixer des limites. Dans quelques articles, ils endossent le rôle d'anti-sujet, interdisant à leur enfant de jouer. Par exemple, le père de Rémi mène « un combat quasi quotidien " (Duretz, 14/05/18) pour que son fils de 8 ans vienne manger au lieu de jouer. D'autres parents « ont interdit [les jeux vidéo au] plus jeune qui perdait pied en classe » (Laronche, 23/01/13). La référence au " combat » traduit l'opposition des programmes narratifs de l'adolescent et du/des parent(s). ${ }^{18}$ Toutefois, quel que soit leur rôle actantiel, c'est souvent l'échec qui domine. Qu'il s'agisse de limiter le temps passé à jouer ou d'interdire les jeux vidéo, les parents font souvent face au refus. Ils se sentent: «impuissants", "désemparés ", "dépassés ", «culpabilisés ", la mère de Johann mène " un désespérant combat », etc. Seuls deux articles relatent le succès de la performance des parents en tant qu'anti-sujets. Après avoir interdit les jeux vidéo, les parents avouent lui «consacr[er] plus de temps et, finalement, son grand frère suit le mouvement et joue moins» (Laronche, 23/01/13). Dans un autre article, Marc témoigne : « son père l'a inscrit dans un internat. "Grâce à cela je m'en suis sorti [...]. Je n'avais plus d'ordinateur, j'étais obligé d'aller en cours. J'ai retrouvé des horaires de travail et une hygiène de vie" "(Laronche, 22/03/10). Enfin, les parents ont parfois le rôle de destinateur d'un nouveau programme narratif, lorsqu'ils prennent rendez-vous pour une consultation : « ce sont en général les parents qui appellent" (Vincent, 4/05/05) ou encore "les demandes de consultations en addictologie pour usage excessif de jeux vidéo proviennent en général de parents inquiets " (Vincent, 3/05/06), etc. En consultant le corps médical, ils enclenchent un nouveau programme narratif pour leur enfant : jouer autrement pour ne plus être dans l'excès.

\section{La figure des pouvoirs publics : un acteur quasi-inexistant}

38 La figure des pouvoirs publics est quasiment absente des discours puisqu'elle n'est mentionnée qu'à deux reprises. Un article évoque une «série de recommandations sur le temps de jeu» (Ducourtieux, 25/12/07) publiée par un organisme auquel sont associés les pouvoirs publics. Ils endossent ici le rôle de l'anti-destinateur, mettant en place des mesures afin d'empêcher que des pratiques problématiques ne surgissent. Dans le second article, deux médecins interpellent les pouvoirs publics : « Il sera alors temps de demander au politique de tenir son rôle de régulateur [...]. Il devra à la fois garantir la cohérence entre le développement économique et l'enrichissement culturel permis par ces nouveaux médias. Il devra aussi être attentif à l'éducation à ces usages, à la prévention et aux soins parfois nécessaires " (Rocheret et Couteron, 19/02/18). Les pouvoirs publics sont placés en position d'anti-destinateur virtuel. En effet, dans ces articles, les pouvoirs publics sont présents indirectement (il n'y a pas d'hommes politiques qui s'expriment dans les pages du Monde) en tant que destinataires des discours d'autres acteurs (ici, la sphère médicale). Il n'y a pas de politisation ${ }^{19}$ de la problématique des pratiques vidéoludiques, la politisation d'un sujet impliquant une présence dudit sujet dans les pages politiques et une place plus marquée de la sphère politique dans le contenu des articles (Azeddine, Blanchard et Poncin, 2007, p. 116-118). Or, dans notre corpus qu'il s'agisse de l'évolution du rubriquage ou de la place de la figure des pouvoirs publics, aucun de ces deux critères ne permet de conclure à une 
politisation des représentations des pratiques vidéoludiques. La presse étudiée ne coconstruit pas ce sujet comme un problème de santé publique.

\section{Conclusion}

L'analyse des discours du Monde sur l'addiction et les pratiques excessives du jeu vidéo entre 1998 et 2019 ne permet pas de conclure à une évolution des représentations. La production discursive est relativement faible et éparse. Le cadrage du sujet est à la fois scientifique, culturel et sociétal, mais il n'est pas construit comme un problème de santé publique, le cadrage politique étant inexistant et la figure des pouvoirs publics quasiment absente. La figure du joueur est plurielle : enfants, adolescents ou encore jeunes adultes. Leurs pratiques oscillent entre usage excessif, dépendance et stabilisation dans la carrière. La représentation des risques est ambivalente : d'un côté, ce sont l'agressivité, la désocialisation et la dépendance qui sont mises en avant; de l'autre, des « experts » soulignent les effets positifs des jeux vidéo. Enfin, la prévention est principalement déléguée aux parents qui endossent à la fois le rôle d'antidestinateur, d'anti-sujet voire de destinateur d'un nouveau programme narratif, lequel est peu détaillé. La gestion des pratiques vidéoludiques est donc renvoyée à la sphère privée. Nous pouvons ajouter que notre analyse des discours du Monde ne corrobore pas complètement certains écrits scientifiques (Mauco, 2008 ; Blanchet, 2008) qui pointent du doigt les médias comme étant à l'origine d'une représentation négative des jeux vidéo. Une étude plus large des productions médiatiques, à la fois audiovisuelles et écrites, permettrait d'affiner le lien entre ces représentations et les lignes éditoriales de différents médias, aux lectorats différents ${ }^{20}$.

\section{BIBLIOGRAPHIE}

AARSETH E. et al. (2016), « Scholars' open debate paper on the World Health Organization ICD-11 Gaming Disorder proposal », Journal of Behavioral Addictions, 3(3), pp. 1-4, https://akademiai.com/ doi/full/10.1556/2006.5.2016.088

AZEDDINE L., BLANCHARD G. et PONCIN C. (2007), « Le cancer dans la presse d'information générale : quelle place pour les malades? », Questions de communication, 11, pp. 111-127.

BAILLY D. (2009), Alcool, drogues chez les jeunes : agissons, Paris, Odile Jacob.

BECKER O. (1985), Outsiders, Paris, Métailié.

BELLEVERGUE S. (2017), « Parents et addiction aux jeux vidéo... de leur enfant : un symptôme écran de la dépression à l'adolescence ? », Dialogue, 217, pp. 71-84.

BLANCHET A. (2008), «Violence, cinéma et jeux vidéo : de la récurrence d'un même discours », Quaderni, 67, http://journals.openedition.org/quaderni/188.

CHABROL C., CHARAUDEAU P. et HOUDEBINE A.-M. (1988), « Préface », in P. Charaudeau (dir.), La presse - production, produit, réception, Paris, Didier Erudition. 
DELFORCE B. (1996), «La responsabilité sociale du journaliste : donner du sens », Les Cahiers du journalisme, 2, pp. 16-32.

DELFORCE B. (2003), « Les spécificités de la médiatisation : l'information scientifique au risque des médias ", in C. Le Boeuf C. et N Pélissier. (dir.), Communiquer l'information scientifique :éthique du journalisme et stratégies des organisations, Paris, L'Harmattan, pp. 163-175

EVENO P. (2004), Histoire du journal "Le Monde" 1944-2004, Paris, Albin Michel.

FAYARD (1993), Sciences aux quotidiens : l'information scientifique et technique dans les quotidiens nationaux européens, Nice, Z'éditions.

FOUCHARD C. (2013), « No life », Cahiers jungiens de psychanalyse, 137, pp. 79-87.

GERSTLE J. (2004), La communication politique, Paris, Armand Colin.

GREIMAS A .-J. (1976), « Préface », in Courtès J., Introduction à la sémiotique narrative et discursive : méthodologie et application, Paris, Hachette, pp. 5-25.

GREIMAS A .-J. (1983), Du sens II : essais sémiotiques, Paris, Seuil.

INSERM (2014), Conduites addictives chez les adolescents. Usage, prévention et accompagnement, Paris, Inserm.

MAUCO O. (2008), « La médiatisation des problématiques de la violence et de l'addiction aux jeux vidéo », Quaderni, 67, http://journals.openedition.org/quaderni/190

MAYAFFRE D. (2002), « Les corpus réflexifs : entre architextualité et hypertextualité », Corpus, 1, http://corpus.revues.org/11.

MISSION INTERMINISTERIELLE DE LUTTE CONTRE LES DROGUES ET LES CONDUITES ADDICTIVES (2018), Plan national de mobilisation contre les addictions (2018-2022), https://www.drogues.gouv.fr/ sites/drogues.gouv.fr/files/atoms/files/plan_mildeca_2018-2022_def_190212_web.pdf

MOIRAND S. (2001), « Du traitement différent de l'intertexte selon les genres convoqués dans les événements scientifiques à caractère politique », Semen, 13, pp. 97-117.

NACHEZ M. et SCHMOLL P. (2003), « Violence et sociabilité dans les jeux vidéos en ligne », Sociétés, 82, pp. 5-17.

NEVEU E. (1999), « L'approche constructiviste des "problèmes publics" - Un aperçu des travaux anglosaxons ", Etudes de Communication, 22, pp. 41-57.

OBRADOVIC I. et al. (2014), « Ecrans et jeux vidéo à l'adolescence », Tendances, 97.

OBRADOVIC I. (2018), « Consultations jeunes consommateurs : bilan après dix ans d'activité », La santé en action, 446, pp. 39-41.

OLIEVENSTEIN C. (2001), Toxicomanie et devenir de l'humanité : le centre médical Marnottan - une expérience française, Paris, Odile Jacob.

SENAT (2008), Le phénomène addictif : mieux le connaître pour mieux le combattre, rapport d'information de Mme Anne-Marie Payet, http://www.senat.fr/rap/r07-487/r07-487_mono.html SYNDICAT DES EDITEURS DE LOGICIELS DE LOISIRS (2018), L'essentiel du jeu vidéo - Chiffres 2017 du marché français du jeu vidéo, https://www.sell.fr/sites/default/files/essentiel-jeu-video/ sell_essentiel_du_jeu_video_2019_0.pdf.

TISSERON S. (2012), « Clinique du virtuel : rêvasser, rêver ou imaginer », Adolescence, 79, pp. 145-157.

TRICLOT M. (2011), Philosophie des jeux vidéos, Mesnil-sur-l'Estrée, Zones. 
VALLEUR M. et VELEA D. (2002), Les addictions sans drogue(s), Toxibase, 6, pp. 1-15.

VALLEUR M. (2006), « L'addiction aux jeux vidéo : une dépendance émergente ? », Enfances \& Psy, 31 , pp. 125-133.

VALLEUR M. (2009), « La cyberaddiction existe-t-elle ? », Psychotropes, 15, pp. 9-19.

VERON E. (1985) «L'analyse du "contrat de lecture" : une nouvelle méthode de positionnement des supports de presse ", in Les médias : expériences et recherches actuelles, Paris, IREP.

\section{Corpus des articles analysés}

AUDUREAU W., « "Fortnite", le jeu vidéo qui rend vos ados accros », Le Monde, 11/04/18

BLANCHARD S., « Une première expertise collective met en garde contre les dangers de l'addiction au jeu », Le Monde, 23/07/08

BRAFMAN N., « Engloutis dans un monde virtuel », Le Monde, 11/05/06

CABUT S., « Le numérique, une "drogue" qui fait débat », Le Monde, 31/01/18

DELAHAYE M., « Strip Tease », Le Monde, 3/08/09

DUCOURTIEUX C., « Les ventes de jeux vidéo égalent celles de musique », Le Monde, 25/12/07

DURETZ M. , « Terrain de jeux », Le Monde, 3/04/13

DURETZ M, « Tu me lâches cette manette ? ! ", Le Monde, 14/05/18

FIÉVET S., « Le virtuel pour soigner les enfants en difficulté », Le Monde, 5/03/08

G. F., « Qui a peur des jeux vidéo ?», Le Monde, 28/09/09

GRANGERAY E., « Enquête d'auteur, "No Pasaràn" de Christian Lehmann », Le Monde, 7/02/2000

KAHN A., « Les Français sont de plus en plus friands de jeux vidéo », Le Monde, 4/05/98

LARONCHE M., « Serge Tisseron : "L'addiction aux jeux vidéo est rare" », Le Monde, 7/01/09

LARONCHE M., «Jeux vidéo : une pratique répandue où les phénomènes de dépendance sont mineurs », Le Monde, 22/03/10

LARONCHE M., « Accro aux jeux vidéo, c'est grave, docteur ? », Le Monde, 11/10/10

LARONCHE M., «Face aux écrans, responsabiliser les enfants plutôt qu'interdire », Le Monde, $23 / 01 / 13$

KERVIEL S., « Entretien : Marcel Rufo », Le Monde, 11/05/09

NORMAND J.-M., « Le retour de Tetris, le jeu vidéo préféré des adultes », Le Monde, 14/09/02

ROCHERET B. et J.-P. COUTERON, « Diaboliser les jeux vidéo est inutile », Le Monde, 19/02/18 ROLLOT C., « Mamie, tu lâches la console ? », Le Monde, 26/10/15

SANTI P., « Les effets pervers d'une fascination », Le Monde, 29/10/14

SANTI P., « Un ado francilien sur huit a un usage problématique des jeux vidéo », Le Monde, $18 / 12 / 14$

VINCENT C., « Jouer pour apprendre à vivre ", Le Monde, 4/05/05

VINCENT C., « Drogués aux jeux virtuels », Le Monde, 3/05/06 
VINCENT C., « Les jeux vidéo, une autre façon de jouer à plusieurs ", Le Monde, 24/05/14

ZILBERTIN O., « L'enfance virtuelle », Le Monde, 2/06/08

\section{NOTES}

1. L'enquête porte sur plus de 2000 élèves scolarisés - de la $4^{\mathrm{e}}$ à la $1^{\text {ère }}$ - dans 15 établissements de la région parisienne.

2. L'actualité scientifique et technique est traitée dans Le courrier des sciences et des techniques qui devient ensuite Le monde des sciences puis le cahier Sciences et médecine (Fayard, 1993, p. 21).

3. Base de données qui répertorie des sources régionales, nationales et internationales en presse généraliste et spécialisée.

4. Dans les années 1970, lorsque la consommation de drogues est devenue un problème public, Claude Olievenstein a proposé cette approche de la toxicomanie à partir de son expérience clinique (Olievenstein, 2001, p.32). Elle permet de comprendre de façon plus fine la consommation de drogues que la distinction drogues dures/drogues douces.

5. " addict* et "jeux vidéo"»; "dépendan* et "jeux vidéo" »; «accro* et "jeux vidéo"»; "souffran* et "jeux vidéo" » dans tout le texte. Ces différentes requêtes nous ont permis de constituer un corpus clos et donc limité à la problématique de l'addiction aux jeux vidéo. Par conséquent, nous n'aborderons pas les autres questions relatives aux pratiques vidéoludiques.

6. Journaliste qui couvre tout ce qui est lié à la psychologie, aux addictions et - dans une moindre mesure - à l'éducation.

7. Elle est également médecin.

8. Sylvie Kerviel, Olivier Zilbertin, Martine Delahaye et Marlène Duretz (journaliste multimédias, auteure de la chronique quotidienne « C'est tout net ! » et du supplément L'Epoque du Monde).

9. Sont évoqués les «adultes qui sont désormais "accros" au jeu» (4/05/98); Tetris, «un jeu entêtant, source d'addictions passagères mais obstinées » qui est aussi le jeu préféré des adultes (14/09/02); des seniors qui pratiquent les jeux vidéo dans le cadre d'un atelier (26/10/15).

10. Respectivement $4,22,8,3,2$ et 41 occurrences.

11. La performance est une phase de transformation. Elle consiste en un «faire-être » qui peut correspondre, dans notre corpus, à la pratique du jeu (Greimas, 1983, p. 70).

12. Elle « renvoie à la suite des passages d'une position à une autre accompli par un travailleur dans un système professionnel » (Becker, 1985, p. 47). Becker recourt à cette notion pour étudier la carrière des fumeurs de marijuana : il existe différentes étapes dans leur carrière (le débutant, l'utilisateur occasionnel et l'utilisateur régulier) et l'entrée dans la carrière n'est pas définitive.

13. World of Warcraft (Blizzard Entertainment, 2004).

14. Un expert dans le champ médiatique n'est pas forcément un expert, dans le champ scientifique. Comme l'explique Delforce, les «figures de savoir ou d'expertise » (Delforce, 2003, p. 170) amenées à s'exprimer dans l'espace public sont plus «floues» et nombreuses que celles qui sont entendues dans le monde de la science car le spécialiste - à même de s'exprimer sur un sujet - n'est pas forcément le même que dans le champ scientifique. Les hiérarchisations de l'espace médiatique ne sont pas celle du champ scientifique.

15. Le terme de « loisir » n'est employé qu'à deux reprises dans l'ensemble du corpus, pour définir le jeu vidéo.

16. Menée par l'Observatoire Français des Drogues et des Toxicomanies, auprès de 2000 élèves de la 4 à la $1^{\text {ère }}$, dans quinze établissements d'île-de-France. L'enquête a été publiée le 17/12/14.

17. Il s'agit d'une référence au système de classification des jeux, par âge (7/01/09). 
18. Pour Greimas, il y a toujours un dédoublement des programmes narratifs, le sujet étant confronté à un anti-sujet qui convoite le même objet que lui ou dont le programme s'oppose au sien.

19. Nous entendons cette notion au sens que lui donne Gerstlé : « le travail qui consiste à affecter à une autorité publique la prise en charge du problème ainsi reconnu publiquement. Politiser une situation, c'est faire admettre que le règlement du problème revient à l'autorité publique quelle qu'elle soit, c'est la reconnaissance de la responsabilité du traitement de la question concernée » (Gerstle, 2004, p. 16)

20. En effet, chaque quotidien - et plus largement média - construit son discours en fonction d'un «contrat de lecture " (Véron, 1985, p. 75) entre l'énonciateur et le destinataire. La figure d'un destinataire idéal préside aux choix de la mise en page, des événements ou encore des thématiques qui vont être couverts par le journal, mais aussi aux représentations et aux mots choisis pour les mettre en récit.

\section{RÉSUMÉS}

Cet article étudie les représentations des pratiques vidéoludiques dans le quotidien national français Le Monde, entre 1998 et 2019. Les jeux vidéo étant intégrés au Plan National contre les Addictions 2018-2022, nous pouvons nous interroger sur les discours tenus par Le Monde à ce sujet. Sur quels types de pratiques le journal se focalise-t-il ? Quelles significations leur sont-elles attribuées? Quelles représentations de la figure du joueur construit le quotidien? Pour répondre à ces questions, nous menons une analyse quantitative et qualitative d'un corpus de 26 articles. L'analyse quantitative permet de caractériser la médiatisation de l'addiction des pratiques vidéoludiques. L'analyse qualitative se fonde sur la sémiotique narrative greimassienne pour étudier les phases du schéma narratif. L'analyse lexicale porte sur les termes employés et les figures construites par le quotidien.

This article examines the representations of the practices of videogame playing in the national French daily newspaper Le Monde, between 1998 and 2019. Video games being integrated into the 2018-2022 Addiction National Plan, we can examine the discourses made by Le Monde about this subject. On what kind of practices does it focus? Which meanings are they given to it? Which representations of the figure of the gamer does the daily newspaper construct? To answer these questions, we do a quantitative and qualitative analysis of a corpus of 26 articles. The quantitative analysis allows to characterize the media coverage of the practices of videogame playing. The qualitative analysis is based on the Greimasian narrative semiotics in order to study the phases of the narrative schema. The lexical analysis considers the words used and the figures constructed by the newspaper.

\section{INDEX}

Mots-clés : jeu vidéo, adolescent, Le Monde, représentations, discours, schéma narratif

Keywords : video game, teenager, Le Monde, representations, discourses, narrative analysis 
AUTEUR

AUDREY ARNOULT

Laboratoire d'Etudes et de Recherches Appliquées en Sciences Sociales, Université Toulouse III Paul Sabatier. 